\title{
Val M. Runge. Imaging of Cerebrovascular Disease. A Practical Guide
}

\author{
Thieme Medical Publishers, New York/Stuttgart, 2017. ISBN: 978-1-62623-248-8
}

\author{
Manuela Amato ${ }^{1} \cdot$ Luigi Mansi $^{2}$
}

Published online: 21 January 2018

(C) Springer-Verlag GmbH Germany, part of Springer Nature 2018

This book seeks to fully explain together with highquality images the complex vascular anatomy and pathology of the central nervous system. The author is Prof. Val M. Runge, from the University Hospital in Bern, and also Editor in Chief of Investigative Radiology. He is an early pioneer in MRI, and known for his work since 1982. He demonstrated for the first time the potential and the early diagnostic utility of intravenous contrast media in MRI, specifically gadolinium chelates.

The book is organized into six chapters. The first chapter deals with recent technological innovations in MRI and $\mathrm{CT}$ with the main focus on topics including dose saving, increase in spatial resolution and artefacts in perfusion cerebrovascular imaging. The second chapter describes normal cerebrovascular anatomy and its main variants, and includes illustrative cases with high-quality $3 \mathrm{D}$ images. The next chapters discuss the most common acute vascular pathologies such as cerebral haemorrhage and ischaemia, explaining the appearance and temporal evolution of these events, focusing on pathological imaging and $\mathrm{CT}$ and MRI representations. The following chapter starts with an introduction to cerebrovascular ischaemia, summarizing causes, clinical features and related deficits. Some easy concepts are explained, such as the importance of imaging in stroke triage; in this regard the use of perfusion CT is particularly discussed. This allows important parameters such as cerebral blood flow to be estimated, giving information on the potentially salvageable cerebral tissue. The last two chapters discuss intracranial aneurysms and other vascular malformations. The chapter includes many cases starting from diagnosis with imaging to treatment with endovascular techniques. The chapters discuss MRI and CT angiography in a very easily understood manner, and are enriched with comparisons between $\mathrm{CT}$ and MRI images obtained with various sequences.

The book is recommended as a valid guide that can be used in daily clinical practice by any doctor interested in the imaging of cerebrovascular disease. Therefore, although it is mainly directed to neuroradiologists, it may be of interest to neurosurgeons, neurologists and residents and students who want to learn more about this important issue.
Luigi Mansi

mansi.luigi@libero.it

1 University Federico II, Naples, Italy

2 Interuniversity Research Center for Sustainability (CIRPS), Naples, Roma, Italy 